\title{
Some Newton-like methods with sharper error estimates for solving operator equations in Banach spaces
}

\author{
DR Sahu*, Krishna Kumar Singh and Vipin Kumar Singh
}

\author{
* Correspondence: \\ drsahudr@gmail.com \\ Department of Mathematics, \\ Faculty of Science, Banaras Hindu \\ University, Varanasi-221005, India
}

\begin{abstract}
It is well known that the rate of convergence of S-iteration process introduced by Agarwal et al. (pp. 61-79) is faster than Picard iteration process for contraction operators. Following the ideas of S-iteration process, we introduce some Newton-like algorithms to solve the non-linear operator equation in Banach space setting. We study the semi-local as well as local convergence analysis of our algorithms. The rate of convergence of our algorithms are faster than the modified Newton method.

Mathematics Subject Classification 2010: 49M15; 65K10; 47H10.

Keywords: Banach contraction theorem, fixed point, Fréchet derivative, Newton's method, nonlinear operator equations, quasi-contraction, S-operator.
\end{abstract}

\section{Introduction}

Let $D$ be an open convex subset of a Banach space $X$ and $F$ be a Fréchet differentiable operator at each point of $D$ with values in a Banach space $Y$. In the sequel, given any $x \in X$ and $r>0, B_{r}[x]$ will designate the set $\{y \in X:\|y-x\| \leq r\}, B_{r}(x)$ will designate the set $\{y \in X:\|y-x\|<r\}, B(Y, X)$ will designate the space of all bounded linear operators from $Y$ to $X$ and $\mathbb{N}_{0}$ will designate the set $\mathbb{N} \cup\{0\}$.

Many applied problems can be formulated to fit the model of the nonlinear operator equation

$$
F(x)=0,
$$

where $F$ is Fréchet differentiable operator at each point of $D$ with values in a Banach space $Y$. A lot of problems about finding the solution of (1.1) are brought forward in many sciences and engineering (see [1]). Undoubtedly, Newton method is the most popular method for solving such problems. Starting with $x_{0} \in X$, the famous Newton method is given by

$$
x_{n+1}=x_{n}-F_{x_{n}}^{\prime-1} F\left(x_{n}\right), \quad n \in \mathbb{N}_{0},
$$

where $F_{x}^{\prime}$ denotes the Fréchet derivative of $F$ at the point $x \in D$. There are numerous generalizations of Newton method for solving nonlinear operator Equation (1.1). Details can be found in Argyros [2], Wu and Zhao [3] and references therein.

(C) 2012 Sahu et al; licensee Springer. This is an Open Access article distributed under the terms of the Creative Commons Attribution License (http://creativecommons.org/licenses/by/2.0), which permits unrestricted use, distribution, and reproduction in any medium, provided the original work is properly cited. 
In Newton method (1.2), functional value of inverse of derivative is required at each iteration. This bring us a natural question how to modify Newton iteration process (1.2), so that the computation of the inverse of derivative at each step in Newton method (1.2) can be avoided. Argyros [4], Bartle [5], Dennis [6] and Rheinboldt [7] discussed the modified Newton method

$$
x_{n+1}=x_{n}-F_{x_{0}}^{-1} F\left(x_{n}\right), \quad n \in \mathbb{N}_{0} .
$$

In [8], Argyros proved the following theorem for semilocal convergence analysis of (1.3) to solve the operator Equation (1.1).

Theorem 1.1 Let $F$ be a Fréchet differentiable operator defined on an open convex subset $D$ of a Banach space $X$ with values in a Banach space $Y$. For some $x_{0} \in D$, let $F_{x_{0}}^{-1} \in B(Y, X)$. Assume that $F_{x_{0}}^{\prime-1}$ and $F$ satisfy the following conditions:

(i) $\left\|F_{x_{0}}^{\prime-1}\right\| \leq \beta$, for some $\beta>0$,

(ii) $\left\|F_{x_{0}}^{\prime-1} F\left(x_{0}\right)\right\| \leq \eta$, for some $\eta>0$,

(iii) $\left\|F_{x}^{\prime}-F_{y}^{\prime}\right\| \leq K_{0}\|x-y\|, \forall x, y \in D$ and for some $K_{0}>0$.

Assume that $h=\eta \beta K_{0}<\frac{1}{2}$ and $B_{r}\left[x_{0}\right] \subseteq D$, where $r=\frac{1-\sqrt{1-2 h}}{h} \eta$. Then we have the following:

(a) The Equation (1.1) has a unique solution $x^{*} \in B_{r}\left[x_{0}\right]$.

(b) The sequence $\left\{x_{n}\right\}$ generated by $(1.3)$ is in $B_{r}\left[x_{0}\right]$ and it converges to $x^{*}$.

(c) The following error estimate holds:

$$
\left\|x_{n+1}-x^{*}\right\| \leq \gamma^{n+1}|| x_{0}-x^{*} \|, \quad \forall n \in \mathbb{N}_{0},
$$

where $\gamma=r \beta K_{0}$.

Let $X$ be a Banach space and $F$ a Fréchet differentiable operator on an open convex subset $D$ of $X$ with values in a Banach space $Y$. Let $x^{*} \in D$ be a solution of (1.1) such that $F_{x^{*}}^{\prime-1} \in B(Y, X)$. For some $x_{0} \in D$, assume that $F_{x^{*}}^{\prime-1}$ and $F$ satisfy the following:

$$
\begin{aligned}
& \left\|F_{x}^{\prime}-F_{x_{0}}^{\prime}\right\| \leq K_{0}\left\|x-x_{0}\right\|, \quad \forall x \in D \text { and for some } K_{0}>0, \\
& \left\|F_{x^{*}}^{\prime-1}\left(F_{x}^{\prime}-F_{x_{0}}^{\prime}\right)\right\| \leq K_{1}\left\|x-x_{0}\right\|, \quad \forall x \in D \text { and for some } K_{1}>0
\end{aligned}
$$

and

$$
\left\|F_{x^{*}}^{\prime-1}\left(F_{x}^{\prime}-F_{x^{*}}^{\prime}\right)\right\| \leq K_{2}\left\|x-x^{*}\right\|, \quad \forall x \in D \text { and for some } K_{2}>0 .
$$

Ren and Argyros [9] studied the following local convergence analysis to solve the operator Equation (1.1).

Theorem 1.2 Let $F$ be a Fréchet differentiable operator defined on an open convex subset $D$ of a Banach space $X$ with values in a Banach space $Y$. Let $X^{*} \in D$ be a solution of (1.1) such that $F_{x^{*}}^{\prime-1} \in B(Y, X)$. For some $x_{0} \in D$, let $F_{x^{*}}^{\prime-1}$ and $F$ satisfy (1.6) and (1.7). Assume that $B_{r_{1}}\left(x^{*}\right) \subseteq D$, where $r_{1}=\frac{2}{K_{2}}$. Then, for any initial point $x_{0} \in B_{r}$ $\left(x^{*}\right)$, where $r=\frac{2}{2 K_{2}+3 K_{1}}$, we have the following: 
(a) The sequence $\left\{x_{n}\right\}$ generated by (1.3) is in $B_{r}\left(x_{0}\right)$ and it converges to the unique solution $x^{*}$ in $B_{r_{1}}\left(x^{*}\right)$.

(b) The following error estimate holds:

$$
\left\|x_{n+1}-x^{*}\right\| \leq\left(\delta_{0}\right)^{n+1}\left\|x_{0}-x^{*}\right\|, \quad \forall n \in \mathbb{N}_{0},
$$

where $\delta_{0}=\frac{\left\|x_{0}-x^{*}\right\|}{r}$.

Recently, Agarwal et al. [10] have introduced the $S$-iteration process as follows: Let $X$ be a normed space, $D$ a nonempty convex subset of $X$ and $A: D \rightarrow D$ an operator. Then, for arbitrary $x_{0} \in D$, the $S$-iteration process is defined by

$$
\left\{\begin{array}{l}
x_{n+1}=\left(1-\alpha_{n}\right) A x_{n}+\alpha_{n} A y_{n} \\
y_{n}=\left(1-\beta_{n}\right) x_{n}+\beta_{n} A x_{n}, \quad n \in \mathbb{N}_{0}
\end{array}\right.
$$

where $\left\{\alpha_{n}\right\}$ and $\left\{\beta_{n}\right\}$ are sequences in $(0,1)$.

In [11], motivated by $S$-iteration process, the first author has introduced the normal $S$-iteration process as follows: Let $X$ be a normed space, $D$ a nonempty convex subset of $X$ and $A: D \rightarrow D$ an operator. Then, for arbitrary $x_{0} \in D$, the normal $S$-iteration process is defined by

$$
x_{n+1}=A\left(\left(1-\alpha_{n}\right) x_{n}+\alpha_{n} A x_{n}\right), \quad n \in \mathbb{N}_{0},
$$

where $\left\{\alpha_{n}\right\}$ be a sequence in $(0,1)$. Noticing that the normal $S$-iteration process is applicable for finding solutions of constrained minimization problems and split feasibility problems (see Sahu [11]).

Following [[11], Theorem 3.6], we remark that the normal $S$-iteration process is faster than the Picard and Mann iteration processes for contraction mappings.

In the present article, motivated by normal $S$-iteration process, we introduce the $S$ iteration processes of Newton-like for finding the solution of operator Equation (1.1).

Algorithm 1.3 Let $\alpha \in(0,1)$. Starting with $x_{0} \in X$ and after $x_{n} \in X$ is defined, we define the next iterate $x_{n+1}$ as follows:

$$
\left\{\begin{array}{l}
x_{n+1}=z_{n}-F_{x_{0}}^{\prime-1} F\left(z_{n}\right), \\
z_{n}=(1-\alpha) x_{n}+\alpha y_{n}, \\
y_{n}=x_{n}-F_{x_{0}}^{\prime-1} F\left(x_{n}\right), \quad n \in \mathbb{N}_{0} .
\end{array}\right.
$$

Algorithm 1.4 Let $\alpha \in(0,1)$. Starting with $x_{0} \in X$ and after $x_{n} \in X$ is defined, we define the next iterate $x_{n+1}$ as follows:

$$
\left\{\begin{array}{l}
x_{n+1}=z_{n}-F_{z_{0}}^{\prime-1} F\left(z_{n}\right), \\
z_{n}=(1-\alpha) x_{n}+\alpha y_{n}, \\
y_{n}=x_{n}-F_{x_{0}}^{\prime-1} F\left(x_{n}\right), \quad n \in \mathbb{N}_{0} .
\end{array}\right.
$$

The purpose of this article is to prove the semi-local as well as local convergence analysis of Algorithms 1.3 and 1.4. It is shown that the rate of convergence of (1.11) and (1.12) are faster than (1.3). Applications to initial value and boundary value problems are included.

\section{Preliminaries}

Definition 2.1 Let $C$ be a nonempty subset of normed space $X$. A mapping $T: C \rightarrow X$ is said to be 
(i) Lipschitzian if there exists a constant $L>0$ such that

$$
\|T(x)-T(y)\| \leq L\|x-y\|, \quad \forall x, y \in C ;
$$

(ii) contraction if there exists a constant $L \in(0,1)$ such that

$$
\|T(x)-T(y)\| \leq L\|x-y\|, \quad \forall x, y \in C ;
$$

(iii) quasi-contraction [12]if there exists a constant $L \in(0,1)$ and

$$
F(T)=\{x \in C: T(x)=x\} \neq \emptyset
$$

such that

$$
\|T(x)-p\| \leq L\|x-p\|, \quad \forall x \in C \text { and } p \in F(T) .
$$

Definition 2.2 [11] Let $C$ be a nonempty convex subset of a normed space $X$ and $T$ : $C \rightarrow C$ an operator. The operator $G: C \rightarrow C$ is said to be S-operator generated by $\alpha \in$ $(0,1)$ and $T$ if

$$
G=T[(1-\alpha) I+\alpha T],
$$

where I is the identity operator.

Before presenting our main results we need the following technical lemmas.

Lemma $2.3[4,13,14]$ Let $P$ be a bounded linear operator on a Banach space X. Then the following are equivalent:

(a) There is a bounded linear operator $Q$ on $X$ such that $Q^{-1}$ exists, and $\|Q-P\|<\frac{1}{\left\|Q^{-1}\right\|}$.

(b) $P^{-1}$ exists.

Further, if $P^{-1}$ exists, then

$$
\left\|P^{-1}\right\| \leq \frac{\left\|Q^{-1}\right\|}{1-\left\|1-Q^{-1} P\right\|} \leq \frac{\left\|Q^{-1}\right\|}{1-\left\|Q^{-1}\right\|\|Q-P\|} .
$$

Lemma 2.4 [9] Let $F$ be a Fréchet differentiable operator defined on an open convex subset $D$ of a Banach space $X$ with values in a Banach space $Y$. Let $x^{*} \in D$ be a solution of (1.1) such that $F_{x^{*}}^{\prime-1} \in B(Y, X)$ and the operator $F$ satisfies the conditions (1.7). Assume that $B_{r}\left(x^{*}\right) \subseteq D$, where $r=\frac{1}{K_{2}}$. Then, for any $x \in B_{r}\left(x^{*}\right), F_{x}^{\prime}$ is invertible, and the following estimate holds:

$$
\left\|\left(F_{x^{*}}^{\prime-1} F_{x}^{\prime}\right)^{-1}\right\| \leq \frac{1}{1-K_{2}\left\|x-x^{*}\right\|} .
$$

Lemma $2.5[15,16]$ Let $(X, d)$ be a complete metric space and $F: X \rightarrow X$ a contraction mapping. Then $F$ has a unique fixed point in $X$.

Lemma 2.6 [[17], Theorem 9.4.2] Let F be a Fréchet differentiable operator defined on an open convex subset $D$ of a Banach space $X$ with values in a Banach space $Y$. Then, for all $x, y \in D$, we have

$$
F(x)-F(y)=\int_{0}^{1} F_{y+t(x-y)}^{\prime}(x-y) d t .
$$




\section{Convergence analysis for Algorithms $\mathbf{1 . 3}$ and $\mathbf{1 . 4}$}

Before studying convergence analysis of Algorithm 1.3, we establish the following theorem for existence of a unique solution of operator Equation (1.1).

Theorem 3.1 Let $F$ be a Fréchet differentiable operator defined on an open convex subset $D$ of a Banach space $X$ with values in a Banach space $Y$. For some $x_{0} \in D$, let $F_{x_{0}}^{\prime-1} \in B(Y, X)$ and the operator $F$ satisfies (1.5) and the following conditions:

(i) $\left\|F_{x_{0}}^{\prime-1} F\left(x_{0}\right)\right\| \leq \eta$, for some $\eta>0$,

(ii) $\left\|F_{x_{0}}^{\prime-1}\right\| \leq \beta$, for some $\beta>0$.

Assume that $h=\eta \beta K_{0}<\frac{1}{2}$ and $B_{r}\left[x_{0}\right] \subseteq D$, where $r=\frac{1-\sqrt{1-2 h}}{h} \eta$. Then, for fixed $\alpha \in$ $(0,1)$, we have the following:

(a) The operator $A: B_{r}\left[x_{0}\right] \rightarrow X$ defined by

$$
A x=x-F_{x_{0}}^{\prime-1} F(x), \quad x \in B_{r}\left[x_{0}\right]
$$

is a contraction self-operator on $B_{r}\left[x_{0}\right]$ with Lipschitz constant $\beta_{r} K_{0}$ and the operator Equation (1.1) has a unique solution in $B_{r}\left[x_{0}\right]$.

(b) The S-operator $A_{\alpha}: B_{r}\left[x_{0}\right] \rightarrow X$ generated by $\alpha$ and $A$ is a contraction self-operator on $B_{r}\left[x_{0}\right]$ with Lipschitz constant $\beta r K_{0}\left(1-\alpha+\alpha \beta r K_{0}\right)$.

Proof: (a) Set $\gamma:=\beta r K_{0}$. Note that $\gamma=1-\sqrt{1-2 h}<1$. For $x, y \in B_{r}\left[x_{0}\right]$, we have

$$
\begin{aligned}
\|A x-A y\| & =\left\|x-y-F_{x_{0}}^{\prime-1}(F(x)-F(y))\right\| \\
& =\left\|F_{x_{0}}^{\prime-1}\left[\int_{0}^{1} F_{\gamma+t(x-\gamma)}^{\prime}(x-y) d t-\int_{0}^{1} F_{x_{0}}^{\prime}(x-\gamma) d t\right]\right\| \\
& \leq\left\|F_{x_{0}}^{\prime-1}\right\| \int_{0}^{1}\left\|F_{y+t(x-\gamma)}^{\prime}(x-\gamma)-F_{x_{0}}^{\prime}(x-\gamma)\right\| d t \\
& \leq \beta \int_{0}^{1} K_{0}\left\|\gamma+t(x-\gamma)-x_{0}\right\|\|x-\gamma\| d t \\
& \leq \beta r K_{0}\|x-\gamma\| \\
& \leq \gamma\|x-\gamma\| .
\end{aligned}
$$

Therefore, the operator $A$ is a contraction with Lipschitz constant $\gamma$. Now we claim that $A\left(B_{r}\left[x_{0}\right]\right) \subseteq B_{r}\left[x_{0}\right]$. For $x \in B_{r}\left[x_{0}\right]$, we have

$$
\begin{aligned}
\left\|A x-x_{0}\right\| & \leq\left\|A x-A x_{0}\right\|+\left\|A x_{0}-x_{0}\right\| \\
& \leq\left\|F_{x_{0}}^{\prime-1}\right\| \int_{0}^{1}\left\|F_{x_{0}}^{\prime}-F_{x_{0}+t\left(x-x_{0}\right)}^{\prime}\right\|\left\|x-x_{0}\right\| d t+\eta \\
& \leq \beta \int_{0}^{1} K_{0}\left\|t\left(x-x_{0}\right)\right\|\left\|x-x_{0}\right\| d t+\eta \\
& \leq \beta K_{0}\left\|x-x_{0}\right\|^{2} \int_{0}^{1} t d t+\eta \\
& \leq \frac{\beta r^{2} K_{0}}{2}+\eta \\
& =\frac{\beta K_{0}}{2}\left[\frac{1-\sqrt{1-2 h}}{h} \eta\right]^{2}+\eta \\
& =r .
\end{aligned}
$$


Hence, the operator $A$ maps $B_{r}\left[x_{0}\right]$ into itself. By Banach contraction principle (see Lemma 2.5), $A$ has a unique fixed point in $B_{r}\left[x_{0}\right]$.

(b) For $x, y \in B_{r}\left[x_{0}\right]$, we have

$$
\begin{aligned}
\left\|A_{\alpha} x-A_{\alpha} y\right\| & =\|A[(1-\alpha) x+\alpha A x]-A[(1-\alpha) y+\alpha A y]\| \\
& \leq \gamma\|(1-\alpha) x+\alpha A x-(1-\alpha) y-\alpha A y\| \\
& \leq \gamma\|(1-\alpha)(x-\gamma)+\alpha(A x-A y)\| \\
& \leq \gamma[(1-\alpha)+\alpha \gamma]\|x-\gamma\| .
\end{aligned}
$$

Therefore, the $S$-operator $A_{\alpha}$ generated by $\alpha$ and $A$ is a contraction operator on $B_{r}$ $\left[x_{0}\right]$ with Lipschitz constant $\beta r K_{0}\left(1-\alpha+\alpha \beta r K_{0}\right)$.

Next we formulate that the operator $A_{\lambda}$ defined by (3.2) is quasi-contraction.

Theorem 3.2 Let $F$ be a Fréchet differentiable operator defined on an open convex subset $D$ of a Banach space $X$ with values in a Banach space $Y$. Assume that $\lambda \in(0,1]$ and $x^{* *} \in D$ is a solution of (1.1) such that $F_{x^{*}}^{\prime-1} \in B(Y, X)$. For some $x_{0} \in D$, let $F_{x^{*}}^{\prime-1}$ and $F$ satisfy the conditions (1.6) and (1.7). Assume that $B_{r_{1}}\left(x^{*}\right) \subseteq D$, where $r_{1}=\frac{2}{K_{2}}$. For $x_{0} \in B_{r}\left(x^{*}\right)$ with $r=\frac{2}{2 K_{2}+3 K_{1}}$, let $A_{\lambda}$ be an operator defined by

$$
A_{\lambda}(x)=x-\lambda F_{x_{0}}^{\prime-1} F(x), \quad \forall x \in B_{r}\left(x^{*}\right) .
$$

Then, we have the following

(a) For $x \in B_{r}\left(x^{*}\right)$, we have

$$
\left\|A_{\lambda}(x)-x^{*}\right\| \leq\left(\lambda \delta_{x}+1-\lambda\right)\left\|x-x^{*}\right\|
$$

where

$$
\delta_{x}=\frac{K_{1}}{2\left(1-r K_{2}\right)}\left(\left\|x-x^{*}\right\|+2\left\|x_{0}-x^{*}\right\|\right) .
$$

(b) $A_{\lambda}$ is a quasi-contraction and self-operator on $B_{r}\left(x^{*}\right)$ with constant $1-(1-\delta) \lambda$, where $\delta=\sup _{x \in B_{r}\left(x^{*}\right)}\left\{\delta_{x}\right\}$.

Proof: (a) For $x \in B_{r}\left(x^{*}\right)$ with $x \neq x^{*}$, we have

$$
\begin{aligned}
\left\|A_{\lambda} x-x^{*}\right\| & =\left\|x-\lambda F_{x_{0}}^{\prime-1} F(x)-x^{*}\right\| \\
& =\left\|\lambda\left(x-x^{*}\right)-\lambda F_{x_{0}}^{\prime-1}\left(F(x)-F\left(x^{*}\right)\right)-(1-\lambda)\left(x-x^{*}\right)\right\| \\
& \leq \lambda\left\|F_{x_{0}}^{\prime-1}\left[F(x)-F\left(x^{*}\right)-F_{x_{0}}^{\prime}\left(x-x^{*}\right)\right]\right\|+(1-\lambda)\left\|x-x^{*}\right\| \\
& =\lambda\left\|\left(F_{x^{*}}^{\prime-1} F_{x_{0}}^{\prime}\right)^{-1} \int_{0}^{1} F_{x^{*}}^{\prime-1}\left(F_{t x+(1-t) x^{*}}^{\prime}\left(x-x^{*}\right)-F_{x_{0}}^{\prime}\left(x-x^{*}\right)\right) d t\right\|+(1-\lambda)\left\|x-x^{*}\right\| \\
& \leq \lambda\left\|\left(F_{x^{*}}^{\prime-1} F_{x_{0}}^{\prime}\right)^{-1}\right\| \int_{0}^{1}\left\|F_{x^{*}}^{\prime-1}\left(F_{t x+(1-t) x^{*}}^{\prime}-F_{x_{0}}^{\prime}\right)\right\|\left\|x-x^{*}\right\| d t+(1-\lambda)\left\|x-x^{*}\right\| .
\end{aligned}
$$

By (1.6) and Lemma 2.4, we have

$$
\begin{aligned}
\left\|A_{\lambda} x-x^{*}\right\| & \leq \frac{\lambda K_{1}}{1-K_{2}\left\|x_{0}-x^{*}\right\|} \int_{0}^{1}\left\|t\left(x-x^{*}\right)+\left(x^{*}-x_{0}\right)\right\|\left\|x-x^{*}\right\| d t+(1-\lambda)\left\|x-x^{*}\right\| \\
& \leq \frac{\lambda K_{1}}{1-K_{2}\left\|x_{0}-x^{*}\right\|} \int_{0}^{1}\left(t\left\|x-x^{*}\right\|+\left\|x_{0}-x^{*}\right\|\right)\left\|x-x^{*}\right\| d t+(1-\lambda)\left\|x-x^{*}\right\| \\
& =\frac{\lambda K_{1}}{1-K_{2}\left\|x_{0}-x^{*}\right\|} \frac{\left(\left\|\left(x-x^{*}\right)\right\|+\left\|x_{0}-x^{*}\right\|\right)^{2}-\left\|x_{0}-x^{*}\right\|^{2}}{2}+(1-\lambda)\left\|x-x^{*}\right\| \\
& \leq \frac{\lambda K_{1}}{2\left(1-r K_{2}\right)}\left(\left\|x-x^{*}\right\|+2\left\|x_{0}-x^{*}\right\|\right)\left\|x-x^{*}\right\|+(1-\lambda)\left\|x-x^{*}\right\| \\
& \leq\left(\lambda \delta_{x}+1-\lambda\right)\left\|x-x^{*}\right\| .
\end{aligned}
$$


(b) The operator $A_{\lambda}$ is a quasi-contraction with constant 1 - $(1-\delta) \lambda$. Indeed,

$$
\begin{aligned}
\sup _{x \in B_{r}\left(x^{*}\right)}\left\{\delta_{x}\right\} & =\frac{K_{1}}{2\left(1-r K_{2}\right)}\left(\sup _{x \in B_{r}\left(x^{*}\right)}\left\|x-x^{*}\right\|+2\left\|x_{0}-x^{*}\right\|\right) \\
& \leq \frac{K_{1}}{2\left(1-r K_{2}\right)}\left(r+2\left\|x_{0}-x^{*}\right\|\right) \\
& <\frac{3 r K_{1}}{2\left(1-r K_{2}\right)} \\
& =1 .
\end{aligned}
$$

This completes the proof. $\square$

Corollary 3.3 Let $F$ be a Fréchet differentiable operator defined on an open convex subset $D$ of a Banach space $X$ with values in a Banach space $Y$. Let $\alpha \in(0,1)$ and $x^{*} L$ $D$ be a solution of $(1.1)$ such that $F_{x^{*}}^{\prime-1} \in B(Y, X)$. For $x_{0} \in D$, let $F_{x^{*}}^{\prime-1}$ and $F$ satisfy the conditions (1.6) and (1.7). Assume that $B_{r_{1}}\left(x^{*}\right) \subseteq D$, where $r_{1}=\frac{2}{K_{2}}$. For $x_{0}$ in $B_{r}\left(x^{*}\right)$ with $r=\frac{2}{2 K_{2}+3 K_{1}}$, let $A$ be an operator defined by (3.1) and let $A_{\alpha}$ be the S-operator generated by $\alpha$ and $A$. Then, the following hold:

$$
\begin{aligned}
& \left\|A(x)-x^{*}\right\| \leq \delta_{x}\left\|x-x^{*}\right\|, \quad \forall x \in B_{r}\left(x^{*}\right), \\
& \left\|A_{\alpha}(x)-x^{*}\right\| \leq \delta_{x}\left(1-\alpha+\alpha \delta_{x}\right)\left\|x-x^{*}\right\|, \quad \forall x \in B_{r}\left(x^{*}\right),
\end{aligned}
$$

where $\delta_{x}$ is defined in (3.4).

Corollary 3.4 Let $F$ be a Fréchet differentiable operator defined on an open convex subset $D$ of a Banach space $X$ with values in a Banach space $Y$. Let $x^{*} \in D$ be a solution of (1.1) such that $F_{x^{*}}^{\prime-1} \in B(Y, X)$. For $x_{0} \in D$ and $\alpha \in(0,1)$, let $F_{x^{*}}^{\prime-1}$ and $F$ satisfy the conditions (1.6) and (1.7). Assume that $B_{r_{1}}\left(x^{*}\right) \subseteq D$, where $r_{1}=\frac{2}{K_{2}}$. For $x_{0}$ in $B_{r}\left(x^{*}\right)$ with $r=\frac{2}{2 K_{2}+3 K_{1}}$, let $U_{\alpha}$ be an operator defined by

$$
U_{\alpha}(x)=x-\alpha F_{x_{0}}^{\prime-1} F(x), \quad \forall x \in B_{r}\left(x^{*}\right) .
$$

Then $U_{\alpha}$ is a quasi-contraction, self-operator on $B_{r}\left(x^{*}\right)$ and the following holds:

$$
\left\|U_{\alpha}(x)-x^{*}\right\| \leq\left(\alpha \delta_{x}+1-\alpha\right)\left\|x-x^{*}\right\|, \quad \forall x \in B_{r}\left(x^{*}\right)
$$

Now, we ready to study the semilocal convergence analysis of Algorithm 1.3.

Theorem 3.5 Let $F$ be a Fréchet differentiable operator defined on an open convex subset $D$ of a Banach space $X$ with values in a Banach space $Y$. For some $x_{0} \in D$, let $F_{x_{0}}^{\prime-1} \in B(Y, X)$. Assume that $F_{x_{0}}^{\prime-1}$ and $F$ satisfy (1.5) with the following conditions:

(i) $\left\|F_{x_{0}}^{\prime-1} F\left(x_{0}\right)\right\| \leq \eta$, for some $\eta>0$,

(ii) $\left\|F_{x_{0}}^{\prime-1}\right\| \leq \beta$, for some $\beta>0$.

Assume that $\alpha \in(0,1), h=\eta \beta K_{0}<\frac{1}{2}$ and $B_{r}\left[x_{0}\right] \subseteq D$, where $r=\frac{1-\sqrt{1-2 h}}{h} \eta$. Then we have the

following:

(a) The Equation (1.1) has a unique solution $x^{*} \in B_{r}\left[x_{0}\right]$. 
(b) The sequence $\left\{x_{n}\right\}$ generated by Algorithm 1.3 is in $B_{r}\left[x_{0}\right]$ and it converges strongly to $x^{*}$.

(c) The following error estimate holds:

$$
\left\|x_{n+1}-x^{*}\right\| \leq \rho^{n+1}\left\|x_{0}-x^{*}\right\|, \quad \forall n \in \mathbb{N}_{0},
$$

where $\rho=\gamma(1-\alpha+\alpha \gamma)$ and $\gamma=\beta r K_{0}$.

Proof : (a) It follows from Theorem 3.1.

(b) From Algorithm 1.3, we have

$$
\begin{aligned}
\left\|x_{n+1}-x^{*}\right\| & =\left\|A_{\alpha}\left(x_{n}\right)-A_{\alpha}\left(x^{*}\right)\right\| \\
& \leq \gamma[(1-\alpha)+\alpha \gamma]\left\|x_{n}-x^{*}\right\| \\
& \leq \gamma^{n+1}[(1-\alpha)+\alpha \gamma]^{n+1}\left\|x_{0}-x^{*}\right\|, \quad \forall n \in \mathbb{N}_{0} .
\end{aligned}
$$

Therefore, $x_{n} \rightarrow x^{*}$ as $n \rightarrow \infty$.

(c) It follows from (3.10).

Remark 3.6 The condition (1.5) of Theorem 3.5 is weaker assumption than assumption (iii) of Theorem 1.1. Also one can observe from (1.4) and (3.9) that

$$
\rho=\gamma(1-\alpha+\alpha \gamma)<\gamma .
$$

The strict inequality (3.11) shows that the error estimate in Theorem 3.5 is sharper than that of Theorem 1.1.

Now, we study the local convergence analysis for Algorithm 1.3.

Theorem 3.7 Let $F$ be a Fréchet differentiable operator defined on an open convex subset $D$ of a Banach space $X$ with values in a Banach space $Y$. Let $\alpha \in(0,1)$ and let $x^{*} L D$ be a solution of (1.1) such that $F_{x^{*}}^{\prime-1} \in B(Y, X)$. For $x_{0} \in D$, let $F_{x^{*}}^{\prime-1}$ and $F$ satisfy the conditions (1.6) and (1.7). Assume that $B_{r_{1}}\left(x^{*}\right) \subseteq D$, where $r_{1}=\frac{2}{K_{2}}$. Then, we have the following:

(a) For initial $x_{0} \in B_{r}\left(x^{*}\right)$ with $r=\frac{2}{2 K_{2}+3 K_{1}}$, the sequence $\left\{x_{n}\right\}$ generated by Algorithm 1.3 is in $B_{r}\left(x^{*}\right)$ and it converges strongly to the unique solution $x^{*}$ in $B_{r_{1}}\left(x^{*}\right)$.

(b) The following error estimate holds:

$$
\left\|x_{n+1}-x^{*}\right\| \leq\left(\rho^{\prime}\right)^{n+1}\left\|x_{0}-x^{*}\right\|, \quad \forall n \in \mathbb{N}_{0},
$$

where $\rho^{\prime}=\delta_{0}\left(1-\alpha+\alpha \delta_{0}\right)$ and $\delta_{0}=\frac{\left\|x_{0}-x^{*}\right\|}{r}$.

Proof: (a) First we show that $x^{*}$ is unique solution of (1.1) in $B_{r_{1}}\left(x^{*}\right)$. For contradiction, suppose that $y^{*}$ is another solution of (1.1) in $B_{r_{1}}\left(x^{*}\right)$. Then, we have

$$
0=F\left(x^{*}\right)-F\left(y^{*}\right)=\int_{0}^{1} F_{\gamma^{*}+t\left(x^{*}-y^{*}\right)}^{\prime}\left(x^{*}-y^{*}\right) d t .
$$

Define an operator $L$ by

$$
L(h)=\int_{0}^{1} F_{\gamma^{*}+t\left(x^{*}-\gamma^{*}\right)}^{\prime} h d t, \quad \forall h \in X .
$$


Consequently, we have

$$
\begin{aligned}
\left\|I-F_{x^{*}}^{\prime-1} L\right\| & =\left\|\int_{0}^{1} F_{x^{*}}^{\prime-1}\left(F_{x^{*}}^{\prime}-F_{\gamma^{*}+t\left(x^{*}-\gamma^{*}\right)}^{\prime}\right) d t\right\| \\
& \leq \frac{K_{2}}{2}\left\|x^{*}-\gamma^{*}\right\| \\
& <\frac{r_{1} K_{2}}{2}=1 .
\end{aligned}
$$

It follows from Lemma 2.3 that the operator $L$ is invertible and hence, $x^{*}=y^{*}$, a contradiction. Thus, $x^{*}$ is the unique solution of (1.1) in $B_{r_{1}}\left(x^{*}\right)$.

Next, we show that $\left\{x_{n}\right\}$ converges to $x^{*}$. By Corollary 3.3, the operator $A_{\alpha}$ is a quasicontraction self-operator on $B_{r}\left(x^{*}\right)$. Thus, $x_{n} \in B_{r}\left(x^{*}\right), \forall n \in \mathbb{N}_{0}$. Now, we have

$$
\begin{aligned}
\left\|x_{n+1}-x^{*}\right\| & =\left\|A_{\alpha}\left(x_{n}\right)-x^{*}\right\| \\
& \leq \delta_{x_{n}}\left[(1-\alpha)+\alpha \delta_{x_{n}}\right]\left\|x_{n}-x^{*}\right\|, \quad \forall n \in \mathbb{N}_{0},
\end{aligned}
$$

where $\delta_{x}$ is defined by (3.4). Since $\delta_{x_{n}}<1, \forall n \in \mathbb{N}_{0}$, we have

$$
0 \leq\left\|x_{n+1}-x^{*}\right\| \leq\left\|x_{n}-x^{*}\right\| \leq \cdots \leq\left\|x_{0}-x^{*}\right\|, \quad \forall n \in \mathbb{N}_{0} .
$$

By definition of $\delta_{x}$, we have

$$
\begin{aligned}
\delta_{x_{n}} & =\frac{K_{1}}{2\left(1-r K_{2}\right)}\left(\left\|x_{n}-x^{*}\right\|+2\left\|x_{0}-x^{*}\right\|\right) \\
& \leq \frac{3 K_{1}\left\|x_{0}-x^{*}\right\|}{2\left(1-r K_{2}\right)} \\
& \leq \frac{\left\|x_{0}-x^{*}\right\|}{r} \\
& =\delta_{0}, \quad \forall n \in \mathbb{N}_{0} .
\end{aligned}
$$

Thus, by (3.13), we have

$$
\left\|x_{n+1}-x^{*}\right\| \leq\left[\delta_{0}\left(1-\alpha+\alpha \delta_{0}\right)\right]^{n+1}\left\|x_{0}-x^{*}\right\|, \quad \forall n \in \mathbb{N}_{0},
$$

which implies $x_{n} \rightarrow x^{*}$ as $n \rightarrow \infty$.

(b) By (3.14), we get the error estimates.

Remark 3.8 One can observe from (1.8) and (3.12) that

$$
\rho^{\prime}=\delta_{0}\left(1-\alpha+\alpha \delta_{0}\right)<\delta_{0} .
$$

The strict inequality (3.15) shows that the error estimate in Theorem 3.7 is sharper than that of Theorem 1.2.

Before presenting local convergence result for Algorithm 1.4, we need the following theorem:

Theorem 3.9 Let $F$ be a Fréchet differentiable operator defined on an open convex subset $D$ of a Banach space $X$ with values in a Banach space $Y$. Let $x^{*} \in D$ be a solution of (1.1) such that $F_{x^{*}}^{\prime-1} \in B(Y, X)$. For $x_{0} \in D$ and $\alpha \in(0,1)$, let $F_{x^{*}}^{\prime-1}$ and $F$ satisfy the conditions (1.6) and (1.7). Let $B_{r_{1}}\left(x^{*}\right) \subseteq D$ and $y_{0} \in B_{r}\left(x^{*}\right)$, where $r_{1}=\frac{2}{K_{2}}$ and $r=\frac{2}{2 K_{2}+3 K_{1}}$. Assume that 


$$
\left\|F_{x^{*}}^{\prime-1}\left(F_{x}^{\prime}-F_{y_{0}}^{\prime}\right)\right\| \leq K_{3}\left\|x-y_{0}\right\|, \quad \forall x \in D \text { and for some } K_{3}>0
$$

Define an operator $V$ by

$$
V(x)=x-F_{\gamma_{0}}^{\prime-1} F(x), \quad \forall x \in B_{r}\left(x^{*}\right) .
$$

Then, we have the following:

(a) For $x \in B_{r}\left(x^{*}\right)$, we have

$$
\left\|V(x)-x^{*}\right\| \leq \delta_{x}^{\prime}\left\|x-x^{*}\right\|,
$$

where $\delta_{x}^{\prime}=\frac{K_{3}}{2\left(1-r K_{2}\right)}\left(\left\|x-x^{*}\right\|+2\left\|y_{0}-x^{*}\right\|\right), \quad \forall x \in B_{r}\left(x^{*}\right)$.

(b) If $K_{3} \leq K_{1}$, then $V$ is a quasi-contraction and self-operator on $B_{r}\left(x^{*}\right)$ with constant $\delta$, where $\delta^{\prime}=\sup _{x \in B_{r}\left(x^{*}\right)}\left\{\delta_{x}^{\prime}\right\}$.

Proof: (a) For $x \in B_{r}\left(x^{*}\right)$, we have

$$
\begin{aligned}
\left\|V x-x^{*}\right\| & =\left\|x-F_{\gamma_{0}}^{\prime-1} F(x)-x^{*}\right\| \\
& =\left\|F_{\gamma_{0}}^{\prime}-1\left(F(x)-F\left(x^{*}\right)-F_{\gamma_{0}}^{\prime}\left(x-x^{*}\right)\right)\right\| \\
& =\left\|\left(F_{x^{*}}^{\prime}-1 F_{\gamma^{0}}^{\prime}\right)^{-1} \int_{0}^{1} F_{x^{*}}^{\prime}{ }^{-1}\left(F_{x^{*}+t\left(x-x^{*}\right)}^{\prime}\left(x-x^{*}\right)-F^{\prime}{ }_{y 0}\left(x-x^{*}\right)\right) d t\right\| \\
& \leq \frac{K_{3}}{2\left(1-K_{2}\left\|y_{0}-x^{*}\right\|\right)}\left[\left(\left\|x-x^{*}\right\|+\left\|y_{0}-x^{*}\right\|\right)^{2}-\left\|y_{0}-x^{*}\right\|^{2}\right] \\
& \leq \frac{K_{3}}{2\left(1-r K_{2}\right)}\left(\left\|x-x^{*}\right\|+2\left\|y_{0}-x^{*}\right\|\right)\left\|x-x^{*}\right\| \\
& =\delta_{x}^{\prime}\left\|x-x^{*}\right\| .
\end{aligned}
$$

(b) The operator $V$ is a quasi-contraction with constant $\delta^{\prime}$. Indeed,

$$
\begin{aligned}
\sup _{x \in B_{r}\left(x^{*}\right)}\left\{\delta_{x}^{\prime}\right\} & =\frac{K_{3}}{2\left(1-r K_{2}\right)}\left(\sup _{x \in B_{r}\left(x^{*}\right)}\left\|x-x^{*}\right\|+2\left\|y_{0}-x^{*}\right\|\right) \\
& \leq \frac{K_{3}}{2\left(1-r K_{2}\right)}\left(r+2\left\|y_{0}-x^{*}\right\|\right) \\
& <\frac{3 r K_{1}}{2\left(1-r K_{2}\right)} \\
& =1 .
\end{aligned}
$$

That completes the proof. $\square$

Now, we ready to study the local convergence analysis for Algorithm 1.4.

Theorem 3.10 Let $F$ be a Fréchet differentiable operator defined on an open convex subset $D$ of a Banach space $X$ with values in a Banach space $Y$. Let $\alpha \in(0,1)$ and let $x^{*} \in D$ be a solution of $(1.1)$ such that $F_{x^{*}}^{\prime-1} \in B(Y, X)$. Assume that $B_{r_{1}}\left(x^{*}\right) \subseteq D$, where $r_{1}=\frac{2}{K_{2}}$. For any $x_{0} \in B_{r}\left(x^{*}\right)$, where $r=\frac{2}{2 K_{2}+3 K_{1}}$, assume that $F_{x^{*}}^{\prime-1}$ and $F$ satisfy the conditions (1.6), (1.7) and (3.16) and that $K_{3} \leq K_{1}$. Then we have the following:

(a) The sequence $\left\{x_{n}\right\}$ generated by Algorithm 1.4 is in $B_{r}\left(x^{*}\right)$ and it converges strongly to the unique solution $x^{*}$ in $B_{r_{1}}\left(x^{*}\right)$. 
(b) The following error estimate holds:

$$
|| x_{n+1}-x^{*}|| \leq\left(\rho^{\prime \prime}\right)^{n+1}|| x_{0}-x^{*} \|, \quad \forall n \in \mathbb{N}_{0},
$$

where $\rho^{\prime \prime}=\left(\alpha \delta_{0}+1-\alpha\right) \delta_{0}^{\prime}$ and $\delta_{0}^{\prime}=\frac{3 K_{3}}{2\left(1-r K_{2}\right)}\left\|x_{0}-x^{*}\right\|$.

Proof: (a) Similarly, as in Theorem 3.7, $x^{*}$ is the unique solution of (1.1) in $B_{r_{1}}\left(x^{*}\right)$. Again, Corollary 3.4 shows that the operator $U_{\alpha}$ defined by (3.7) is a self-operator on $B_{r}\left[x^{*}\right]$. Hence, from (1.12), we have

$$
y_{0}=U_{\alpha} x_{0} \in B_{r}\left(x^{*}\right) .
$$

It follows from Theorem 3.9 that the operator $V$ defined by (3.17) is a self operator on $B_{r}\left[x^{*}\right]$. Thus, 1.4 can be written as

$$
x_{n+1}=V U_{\alpha}\left(x_{n}\right), \quad \forall n \in \mathbb{N}_{0} .
$$

Since,

$$
\begin{aligned}
\left\|x_{n+1}-x^{*}\right\| & =\left\|V U_{\alpha}\left(x_{n}\right)-x^{*}\right\| \\
& \leq \delta_{\gamma_{n}}^{\prime} \| U_{\alpha}\left(x_{n}\right)-x^{*} \\
& \leq\left(\alpha \delta_{x_{n}}+1-\alpha\right) \delta_{\gamma_{n}}^{\prime}\left\|x_{n}-x^{*}\right\|, \quad \forall n \in \mathbb{N}_{0} .
\end{aligned}
$$

By definition of $\delta_{x}^{\prime}$, we have

$$
\begin{aligned}
\delta_{\gamma_{n}}^{\prime} & =\frac{K_{3}}{2\left(1-r K_{2}\right)}\left(\left\|U_{\alpha}\left(x_{n}\right)-x^{*}\right\|+2\left\|x_{0}-x^{*}\right\|\right) \\
& \leq \frac{K_{3}}{2\left(1-r K_{2}\right)}\left(\left(\alpha \delta_{x_{n}}+1-\alpha\right)\left\|x_{n}-x^{*}\right\|+2\left\|x_{0}-x^{*}\right\|\right) \\
& \leq \frac{K_{3}}{2\left(1-r K_{2}\right)}\left(\left\|x_{0}-x^{*}\right\|+2\left\|x_{0}-x^{*}\right\|\right) \\
& =\frac{3 K_{3}}{2\left(1-r K_{2}\right)}\left\|x_{0}-x^{*}\right\| \\
& =\delta_{0}^{\prime}, \quad \forall n \in \mathbb{N}_{0} .
\end{aligned}
$$

By (3.19), we have

$$
\left\|x_{n+1}-x^{*}\right\| \leq\left(\left(\alpha \delta_{0}+1-\alpha\right) \delta_{0}^{\prime}\right)^{n+1}\left\|x_{0}-x^{*}\right\|, \quad \forall n \in \mathbb{N}_{0},
$$

which implies $x_{n} \rightarrow x^{*}$ as $n \rightarrow \infty$.

(b) From (3.20), we get the error estimates. $\square$

Remark 3.11 One can observe from (1.8) and (3.18) that

$$
\begin{aligned}
\rho^{\prime \prime} & =\left(\alpha \delta_{0}+1-\alpha\right) \delta_{0}^{\prime} \\
& =\frac{3 K_{3}\left(\alpha \delta_{0}+1-\alpha\right)}{2\left(1-r K_{2}\right)}\left\|x_{0}-x^{*}\right\| \\
& \leq \frac{3 K_{1}\left(\alpha \delta_{0}+1-\alpha\right)}{2\left(1-r K_{2}\right)}\left\|x_{0}-x^{*}\right\| \\
& \leq \delta_{0}\left(\alpha \delta_{0}+1-\alpha\right) \\
& =\rho \prime<\delta_{0} .
\end{aligned}
$$

The strict inequality (3.21) shows that the error estimate in Theorem 3.10 is sharper than that of Theorems 1.2 and 3.7 . 


\section{Application to initial and boundary value problems}

Throughout this section, let $C 0[1]$ be the space of real-valued continuous functions defined on the interval $0[1]$ with norm

$$
\|x\|=\max _{0 \leq t \leq 1}|x(t)| .
$$

\subsection{Initial value problem}

Consider initial value problem

$$
\frac{d y(s)}{d s}=f(s, y(s)), y(0)=0, \quad 0 \leq s \leq 1 .
$$

Let $f_{2}^{\prime}(s, y(s))=\frac{\partial}{\partial y} f(s, y(s))$, the partial derivative of $f$ with respect to second component. Assume that $f_{2}^{\prime}(s, y(s))$ exists for all $(s, y(s)) \in S \subseteq 0[1] \times \mathbb{R}$ and

$$
\left\|f_{2}^{\prime}(s, y(s))-f_{2}^{\prime}(s, y(s))\right\| \leq K_{0}\left\|y-y_{0}\right\|, \quad \forall y \in C^{1}[0,1] \text { and for some } K_{0}>0,
$$

where $y_{0} \in C^{1} 0[1]$ and $C^{1} 0[1]$ is the space of all real-valued continuously differentiable functions defined on $0[1]$.

Consider the operator $F: C^{1} 0[1] \rightarrow C 0[1]$ defined by

$$
F(y)(s)=\frac{d y(s)}{d s}-f(s, y(s)) .
$$

Then, solving problem (4.1) is equivalent to solving the Equation (1.1). One can observe that the operator $F$ defined by (4.3) is Fréchet differetiable and its Fréchet derivative is given by

$$
F_{\gamma}^{\prime} h(s)=\frac{d h(s)}{d s}-f_{2}^{\prime}(s, y(s)) h(s), \quad \forall h \in C^{1}[0,1] .
$$

Theorem 4.1 Let $F: C^{1} 0[1] \rightarrow C 0[1]$ be an operator defined by (4.3). For some $y_{0} \in$ $C^{1} 0[1]$, assume that $F_{\gamma_{0}}^{\prime-1}$ exists and $f_{2}^{\prime}(s, y(s))$ satisfies (4.2). Suppose that $h=\frac{K_{0}\left\|F\left(y_{0}\right)\right\|}{\left(1-\theta_{1}\right)^{2}}<\frac{1}{2}$, where $\theta_{1}=\sup _{s \in[0,1]}\left|f_{2}^{\prime}\left(s, y_{0}(s)\right)\right|$. Then, we have the following:

(a) The initial value problem (4.1) has a unique solution $y^{*}$ in $B_{r}\left[y_{0}\right]$, where $r=\frac{1-\sqrt{1-2 h}}{h} \eta$.

(b) For $x_{0}=y_{0}$, the sequence $\left\{x_{n}\right\}$ generated by Algorithm 1.3 is in $B_{r}\left[y_{0}\right]$ and it converges strongly to $y^{*}$.

Proof: Our goal is to find an upper bound for $F_{\gamma_{0}}^{\prime-1}$. Set

$$
F_{\gamma_{0}}^{\prime} h(s)=\frac{d h(s)}{d s}-f_{2}^{\prime}\left(s, y_{0}(s)\right) h(s)=u(s) .
$$

Since $F_{\gamma_{0}}^{\prime-1}$ exists, we can immediately write $h(s)=F_{\gamma_{0}}^{-1} u(s)$ and arrive at the first order linear initial value problem 


$$
\left\{\begin{array}{l}
\frac{d h(s)}{d s}=u(s)+f_{2}^{\prime}\left(s, y_{0}(s)\right) h(s) \\
h(0)=0
\end{array}\right.
$$

It should be noted that problem (4.4) is equivalent to the integral equation of Voltera type of second kind (see [18])

$$
h(s)=\int_{0}^{s}\left[u(\tau)+f_{2}^{\prime}\left(\tau, y_{0}(\tau)\right) h(\tau)\right] d \tau
$$

Again consider a operator $L$ defined by

$$
\operatorname{Lh}(s)=\int_{0}^{s} f_{2}^{\prime}\left(\tau, y_{0}(\tau)\right) h(\tau) d \tau
$$

Clearly, the operator $L$ is linear. We can write

$$
(I-L)(h)(s)=\int_{0}^{s} u(\tau) d \tau
$$

Using max-norm in $C 0[1]$, the operator $L$ is bounded and satisfies $\|L\| \leq \theta_{1}$, where $\theta_{1}=\sup _{s \in[0,1]}\left|f_{2}^{\prime}\left(\tau, y_{0}(\tau)\right)\right|$. Consequently, if $\theta_{1}<1$, then by Lemma 2.3 , the inverse $(I-L)^{-1}$ exists and $\left\|(I-L)^{-1}\right\| \leq \frac{1}{1-\theta_{1}}$. Therefore, $\left\|F_{\gamma_{0}}^{\prime-1}\right\| \leq \frac{1}{1-\theta_{1}}$. Indeed

$$
\left\|F_{\gamma_{0}}^{\prime-1} u\right\|=\max _{s \in[0,1]}\left|F_{\gamma_{0}}^{\prime-1} u(s)\right|=\max _{s \in[0,1]}\left|(I-L)^{-1} \int_{0}^{s} u(\tau) d \tau\right| \leq \frac{1}{1-\theta_{1}}\|u\| .
$$

Next, by (4.2), we have

$$
\left\|F_{y}^{\prime}-F_{y_{0}}^{\prime}\right\| \leq K_{0}\left\|y-y_{0}\right\|, \quad \forall y \in C^{1}[0,1] .
$$

Clearly, $\eta=\frac{\left\|F\left(y_{0}\right)\right\|}{1-\theta_{1}}$ and $\beta=\frac{1}{1-\theta_{1}}$. By assumption, $h=\eta \beta K_{0}<\frac{1}{2}$. Thus, all the assumption of Theorem 3.5 are satisfied. Therefore, Theorem 4.1 follows from Theorem 3.5. $\square$

\subsection{Boundary value problem}

Consider the second-order boundary value problem

$$
\frac{d^{2} y(s)}{d s^{2}}=g(s, y(s)), \quad y(0)=0, \quad y(1)=0 .
$$

Assume that $g_{2}^{\prime}(s, y(s))$ exists for all $(s, y(s)) \in S \subseteq[0,1] \times \mathbb{R}$ and

$$
\left\|g_{2}^{\prime}(s, y(s))-g_{2}^{\prime}\left(s, y_{0}(s)\right)\right\| \leq K_{0}\left\|y-y_{0}\right\|
$$

where $y_{0} \in C^{2} 0[1]$ and $C^{2} 0[1]$ is the space of all real-valued second time continuously differentiable functions defined on $0[1]$. Consider the operator $F: C^{2} 0[1] \rightarrow C 0$ [1] defined by 


$$
F(\gamma)(s)=\frac{d^{2} y(s)}{d s^{2}}-g(s, y(s))
$$

Then, solving problem (4.5) is equivalent to solving the (1.1). As in the case of initial value problem, one can observe that the operator $F$ defined by (4.7) is Fréchet differentiable and its Fréchet derivative at each $y \in C^{2} 0[1]$ is given by

$$
F_{y}^{\prime} h(s)=\frac{d^{2} h(s)}{d s^{2}}-g_{2}^{\prime}(s, y(s)) h(s), \quad \forall h \in C^{2}[0,1], \quad \forall s \in[0,1] .
$$

Theorem 4.2 Let $F: C^{2} 0[1] \rightarrow C 0[1]$ be an operator defined by (4.7). For some $y_{0} \in$ $C^{2} 0[1]$, assume that $F_{\gamma_{0}}^{\prime-1}$ exists and $g_{2}^{\prime}(s, y(s))$ satisfies (4.6). Suppose that $h=\frac{K_{0}\left\|F\left(y_{0}\right)\right\|}{\left(8-\theta_{2}\right)^{2}}<\frac{1}{2}$, where $\theta_{2}=\sup _{s \in[0,1]}\left|g_{2}^{\prime}\left(s, y_{0}(s)\right)\right|$. Then, we have the following:

(a) The boundary value problem (4.5) has a unique solution $y^{*}$ in $B_{r}\left[y_{0}\right]$, where $r=\frac{1-\sqrt{1-2 h}}{h} \eta$.

(b) For $x_{0}=y_{0}$, the sequence $\left\{x_{n}\right\}$ generated by Algorithm 1.3 is in $B_{r}\left[y_{0}\right]$ and it converges strongly to $y^{*}$.

Proof: To prove the theorem it is sufficient to find an upper bound of $\left\|F_{\gamma_{0}}^{\prime-1}\right\|$. Set

$$
F_{\gamma_{0}}^{\prime} h(s)=\frac{d^{2} h(s)}{d s^{2}}-g_{2}^{\prime}\left(s, y_{0}(s)\right) h(s)=u(s),
$$

then $h(s)=F_{\gamma_{0}}^{\prime-1} w(s)$ and we arrive at the linear boundary value problem

$$
\left\{\begin{array}{l}
\frac{d^{2} h(s)}{d s^{2}}=u(s)+g_{2}^{\prime}\left(s, y_{0}(s)\right) h(s) \\
u(0)=u(1)=0
\end{array}\right.
$$

Problem (4.8) may be written in the form of the integral equation of Fredholm type of second kind (see [18]) as

$$
h(s)=-\int_{0}^{1} G(s, \tau)\left[u(\tau)+g_{2}^{\prime}\left(\tau, y_{0}(\tau)\right) h(\tau)\right] d \tau,
$$

where

$$
G(s, \tau)=\left\{\begin{array}{l}
\tau(1-s), s \geq \tau \\
s(1-\tau), s \leq \tau .
\end{array}\right.
$$

Consider the operator $L$ defined by

$$
L(h)(s)=-\int_{0}^{1} G(s, \tau) g_{2}^{\prime}\left(\tau, y_{0}(\tau)\right) h(\tau) d \tau
$$

and consequently, we have

$$
(I-L)(h)(s)=-\int_{0}^{1} G(s, \tau) u(\tau) d \tau .
$$


Using the max norm in $C 0[1]$, the operator $L$ is bounded and

$$
\|L\| \leq \frac{\theta_{2}}{8}, \text { where } \theta_{2}=\sup _{s \in[0,1]}\left|g_{2}^{\prime}(s, y(s))\right| .
$$

By Lemma 2.3, there exists $(I-L)^{-1}$ if $\theta_{2}<8$ and

$$
\left\|(I-L)^{-1}\right\| \leq \frac{8}{8-\theta_{2}} .
$$

Observe that

$$
\left\|F_{\gamma_{0}}^{\prime-1}\right\| \leq \frac{1}{8-\theta_{2}} \text { and }\left\|F_{\gamma_{0}}^{\prime-1} F\left(y_{0}\right)\right\| \leq \frac{\left\|F\left(y_{0}\right)\right\|}{8-\theta_{2}} .
$$

Finally, by (4.6), we have

$$
\left\|F_{y}^{\prime}-F_{\gamma_{0}}^{\prime}\right\| \leq K_{0}\left\|y-y_{0}\right\|, \quad \forall y \in C^{2}[0,1]
$$

Clearly, $\eta=\frac{\left\|F\left(y_{0}\right)\right\|}{8-\theta_{2}}$ and $\beta=\frac{1}{8-\theta_{2}}$. By assumption, $h=\eta \beta K_{0}<\frac{1}{2}$. Thus, all the assumption of Theorem 3.5 are satisfied. Therefore, Theorem 4.2 follows from Theorem 3.5.

\section{Numerical examples}

First, we derive the following corollary from Theorem 3.5.

Corollary 5.1 For $N \in \mathbb{N}$, let $F: \mathbb{R}^{N} \rightarrow \mathbb{R}^{N}$ be a Fréchet differentiable operator at each point of an open convex subset $D$ of $\mathbb{R}^{N}$ defined by

$$
F(x)=\left(f_{1}(x), f_{2}(x), \ldots, f_{N}(x)\right), \forall x=\left(x_{1}, x_{2}, \ldots, x_{N}\right) \in D,
$$

where $f_{i}: \mathbb{R}^{N} \rightarrow \mathbb{R}$, for $i=1,2, \ldots, N$. For some $x_{0} \in D$, assume that the Jacobian matrix $\left[J_{F}\left(x_{0}\right)\right]$ of $F$ at $x_{0}$ defined by

$$
\left[J_{F}\left(x_{0}\right)\right]=\left\{\begin{array}{cccc}
\frac{\partial f_{1}\left(x_{0}\right)}{\partial x_{1}} & \frac{\partial f_{1}\left(x_{0}\right)}{\partial x_{2}} & \cdots & \frac{\partial f_{1}\left(x_{0}\right)}{\partial x_{N}} \\
\frac{\partial f_{2}\left(x_{0}\right)}{\partial x_{1}} & \frac{\partial f_{2}\left(x_{0}\right)}{\partial x_{2}} & \cdots & \frac{\partial f_{2}\left(x_{0}\right)}{\partial x_{N}} \\
\cdots & \cdots & \cdots & \cdots \\
\frac{\partial f_{N}\left(x_{0}\right)}{\partial x_{1}} & \frac{\partial f_{N}\left(x_{0}\right)}{\partial x_{2}} & \cdots & \frac{\partial f_{N}\left(x_{0}\right)}{\partial x_{N}}
\end{array}\right\}
$$

is invertible. Suppose that the inverse matrix $\left[J_{F}\left(x_{0}\right)\right]^{-1}$ and F satisfy the following conditions:

(i) $\left\|\left[U_{F}\left(\boldsymbol{x}_{0}\right)\right]^{-1} F\left(\boldsymbol{x}_{0}\right)\right\| \leq \eta$, for some $\eta>0$,

(ii) $\left.\| U_{F}\left(x_{0}\right)\right]^{-1} \| \leq \beta$, for some $\beta>0$,

(iii) $\left.\left.\| U_{F}(\boldsymbol{x})\right]-U_{F}\left(\boldsymbol{x}_{0}\right)\right]\left\|\leq K_{0}\right\| \boldsymbol{x}-\boldsymbol{x}_{0} \|, \forall \boldsymbol{x} \in D$ and for some $K_{0}>0$.

Let $\alpha \in(0,1), h=\eta \beta K_{0}<\frac{1}{2}$ and $B_{r}\left[x_{0}\right] \subseteq D$, where $r=\frac{1-\sqrt{1-2 h}}{h} \eta$. Then we have the following:

(a) The Equation (1.1) has a unique solution $\boldsymbol{x}^{*} \in B_{r}\left[\boldsymbol{x}_{0}\right]$. 
(b) The sequence $\left\{\boldsymbol{x}_{n}\right\}$ generated by

$$
\left\{\begin{array}{l}
x_{n+1}=z_{n}-\left[J_{F}\left(x_{0}\right)\right]^{-1} F\left(z_{n}\right), \\
z_{n}=(1-\alpha) x_{n}+\alpha y_{n^{\prime}} \\
y_{n}=x_{n}-\left[J_{F}\left(x_{0}\right)\right]^{-1} F\left(x_{n}\right), \quad n \in \mathbb{N}_{0}
\end{array}\right.
$$

is in $B_{r}\left[\boldsymbol{x}_{0}\right]$ and it converges to $x^{*}$.

(c) The following error estimate holds:

$$
\left\|x_{n+1}-x^{*}\right\| \leq \rho^{n+1}\left\|x_{0}-x^{*}\right\|, \quad \forall n \in \mathbb{N}_{0},
$$

where $\rho=\gamma(1-\alpha+\alpha \gamma)$ and $\gamma=\beta r K_{0}$.

Following example shows numerically that (5.1) is faster than the modified Newton method defined by (1.3).

Example 5.2 Let $X=\mathbb{R}, D=(-1,1)$ and $F: D \rightarrow \mathbb{R}$ an operator defined by

$$
F(x)=e^{x}-1, \quad \forall x \in D .
$$

Then $F$ is Fréchet differentiable and its Fréchet derivative $F_{x}^{\prime}$ at any point $x \in D$ is given by

$$
F_{x}^{\prime}=e^{x}
$$

For $x_{0}=0.26$, we have

$$
F_{x_{0}}^{\prime}{ }^{-1}=\frac{1}{e^{0.26}} .
$$

Set $\beta=0.771051585803566, \eta=0.228948414196434$ and $K_{0}=2.718281828459046$, we have $h=\eta \beta K_{0}<\frac{1}{2}$ and

$$
\begin{aligned}
\left\|F_{x_{0}}^{\prime}-1\right\| & \leq \beta_{0}, \\
\left\|F_{x_{0}}^{\prime}-1 F\left(x_{0}\right)\right\| & \leq \eta_{0}, \\
\left\|F_{x}^{\prime}-F_{x_{0}}^{\prime}\right\| & \leq K_{0}\left\|x-x_{0}\right\| .
\end{aligned}
$$

Hence, all the condition of Corollary 5.1 are satisfied. Therefore, the sequence $\left\{x_{n}\right\}$ generated by (5.1) is in $B_{r}\left[x_{0}\right]$ and it converges to a unique $x^{*} \in B_{r}\left[x_{0}\right]$. The Figure 1 and Table 1 show that sequence $\left\{x_{n}\right\}$ generated by (5.1) is faster than the modified Newton method defined by (1.3).

For $N=2$ in Corollary 5.1, the following example shows numerically the convergence of (5.1).

Example 5.3 Let $D=X=Y=\mathbb{R}^{2}$ under the norm

$$
\|x\|=\sqrt{x^{2}+y^{2}}, \quad \forall x=(x, y) \in \mathbb{R}^{2}
$$

and induced matrix norm. Consider an operator $F: \mathbb{R}^{2} \rightarrow \mathbb{R}^{2}$ defined by

$$
F(x)=\left(-x^{2}+\frac{1}{3},-y^{2}+\frac{1}{3}\right), \quad \forall x=(x, y) \in \mathbb{R}^{2} .
$$




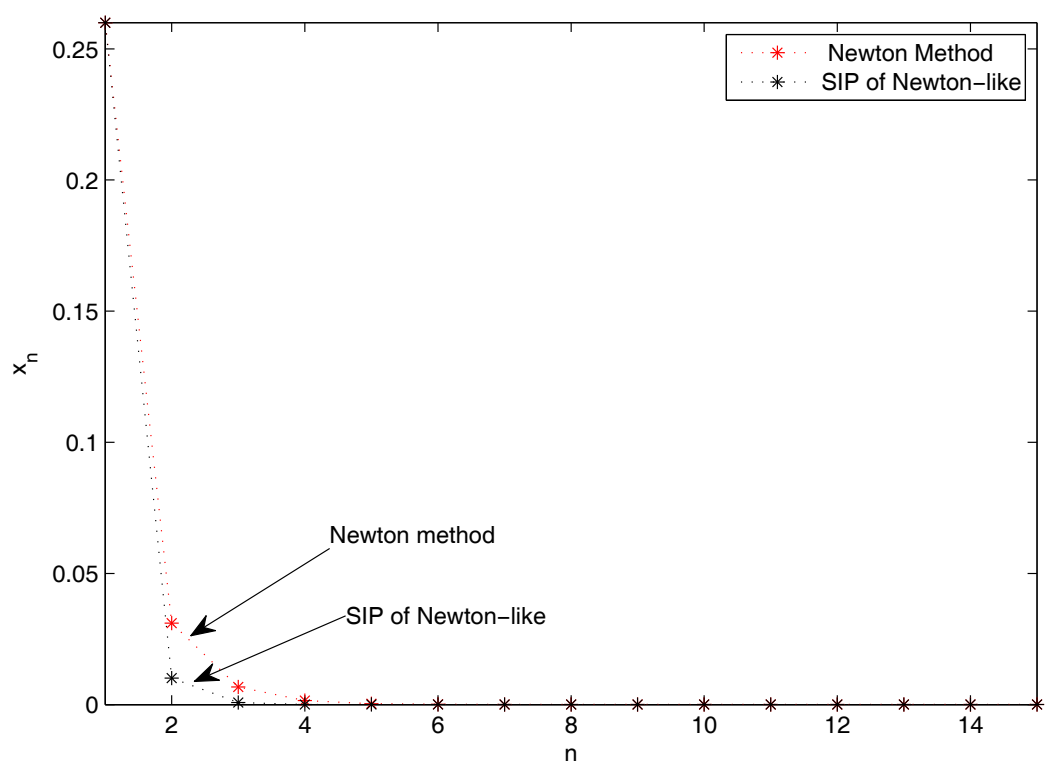

Figure 1 Comparison of the iteration processes defined by (1.3) and (1.11).

Clearly, the point $\left(\frac{1}{\sqrt{3}}, \frac{1}{\sqrt{3}}\right)$ is the zero of $F$ in $D$. It can be seen that $F$ is Fréchet differentiable at each point of $D$ and its Jacobian matrix $\left[J_{F}(x)\right]$ at any point $\boldsymbol{x}=(x, y) \in$ $\mathbb{R}^{2}$ is given by

$$
\left[J_{F}(x)\right]=\left[\begin{array}{cc}
-2 x & 0 \\
0 & -2 y
\end{array}\right] .
$$

Now, for any $\boldsymbol{x}, \boldsymbol{x}_{0} \in D$, we have

$$
\left\|F_{x}^{\prime}-F_{x_{0}}^{\prime}\right\| \leq 2\left\|x-x_{0}\right\| .
$$

For $x_{0}=(1,1)$, we get

$$
\left[J_{F}\left(x_{0}\right)\right]^{-1}=\left[\begin{array}{cc}
-\frac{1}{2} & 0 \\
0 & -\frac{1}{2}
\end{array}\right] \text {. }
$$

Therefore, for $\beta=\frac{1}{\sqrt{2}}, \eta=\frac{\sqrt{2}}{3}$ and $K_{0}=2$, we have $h=\beta \eta K_{0}<1 / 2$ and

$$
\begin{aligned}
\left\|\left[J_{F}\left(x_{0}\right)\right]^{-1}\right\| & \leq \beta, \\
\left\|\left[J_{F}\left(x_{0}\right)\right]^{-1} F\left(x_{0}\right)\right\| & \leq \eta,
\end{aligned}
$$

and $B_{r}\left[x_{0}\right]$, where $r=\frac{1-\sqrt{ }(1-2 h)}{h} \eta=3-\sqrt{3}$, is contained in $D=X$. Hence, all the conditions of Corollary 5.1 are satisfied. One can write sequence $\left\{\boldsymbol{x}_{n}\right\}$ generated by (5.1) as

$$
\boldsymbol{x}_{n+1}=\left[\begin{array}{l}
x_{n+1} \\
y_{n+1}
\end{array}\right]=\left[\begin{array}{l}
\left(\frac{1}{3}-\frac{\alpha^{2}}{72}\right)+\left(1-\frac{\alpha}{6}\right) x_{n}-\left(\frac{1}{2}+\frac{\alpha}{2}-\frac{\alpha^{2}}{12}\right) x_{n}^{2}+\frac{\alpha}{2} x_{n}^{3}-\frac{\alpha^{2}}{8} x_{n}^{4} \\
\left(\frac{1}{3}-\frac{\alpha^{2}}{72}\right)+\left(1-\frac{\alpha}{6}\right) y_{n}-\left(\frac{1}{2}+\frac{\alpha}{2}-\frac{\alpha^{2}}{12}\right) y_{n}^{2}+\frac{\alpha}{2} y_{n}^{3}-\frac{\alpha^{2}}{8} y_{n}^{4}
\end{array}\right] .
$$


Table 1 Comparison of the iteration processes defined by ( 1

\begin{tabular}{llllll}
\hline $\boldsymbol{n}$ & Newton method & SIP of Newton-like & $\boldsymbol{n}$ & Newton method & SIP of Newton-like \\
\hline 1 & 0.2600000000000000 & 0.2600000000000000 & 9 & 0.000000955573754 & 0.000000000272069 \\
2 & 0.031051585803566 & 0.010093382981211 & 10 & 0.000000218776743 & 0.000000000022642 \\
3 & 0.006733609382298 & 0.000820742954637 & 11 & 0.000000050088570 & 0.000000000001884 \\
4 & 0.001524129572619 & 0.000068174499880 & 12 & 0.000000011467698 & 0.0000000000000157 \\
5 & 0.000348051028303 & 0.000005672583045 & 13 & 0.000000002625511 & 0.0000000000000013 \\
6 & 0.000079639023162 & 0.000000472064696 & 14 & 0.000000000601107 & 0.0000000000000001 \\
7 & 0.000018230782847 & 0.000000039285051 & 15 & 0.000000000137622 & 0.0000000000000000 \\
8 & 0.000004173780688 & 0.000000003269291 & 16 & 0.000000000031508 & 0.0000000000000000 \\
\hline
\end{tabular}

Therefore, in view of Corollary 5.1, the sequence $\left\{\boldsymbol{x}_{n}\right\}$ is in $B_{r}\left[\boldsymbol{x}_{0}\right]$ and it converges to a unique $\boldsymbol{x}^{*}=\left(x^{*}, y^{*}\right) \in B_{r}\left[\boldsymbol{x}_{0}\right]$. The Figure 2 and Table 2 show that sequence $\left\{\boldsymbol{x}_{n}\right\}$ generated by (5.1) with $\alpha=0.5$ is faster than the modified Newton method defined by (1.3).

Now, we study the convergence of (1.11) for infinite dimensional cases,

Example 5.4 Let $X=D=C 0[1]$ be the space of real-valued continuous functions defined on the interval $0[1]$ with norm

$$
\|x\|=\max _{0 \leq t \leq 1}|x(t)| .
$$

Consider the integral equation $F(x)=0$, where

$$
F(x)(s)=-1+x(s)+\pi x(s) \int_{0}^{1} \frac{s}{s+t} x(t) d t,
$$

with $s \in 0[1], x \in C 0[1]$ and $\pi \in(0,2]$. Integral equations of this kind called Chandrasekhar equations arise in elasticity or neutron transport problems [19]. The norm is taken as sup-norm. Now it is easy to find the Fréchet derivative of $F$ as

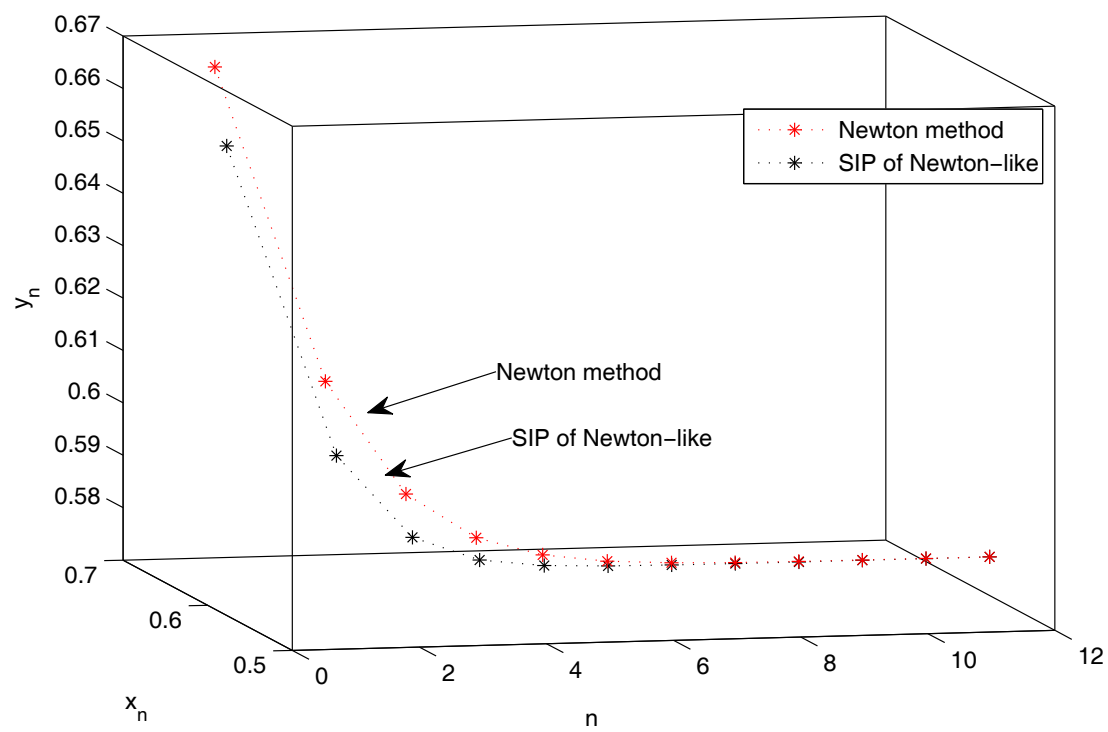

Figure 2 Comparison of the iteration processes defined by (1.3) and (5.1). 
Table 2 Comparison of the iteration processes defined by ( 1

\begin{tabular}{llllll}
\hline $\boldsymbol{N}$ & Newton method & SIP of Newton-like & $\boldsymbol{n}$ & Newton method & SIP of Newton-like \\
\hline 1 & 1 & 1 & 7 & 0.578358062815874 & 0.577513680188369 \\
& 1 & 1 & & 0.578358062815874 & 0.577513680188369 \\
2 & 0.6666666666666667 & 0.652777777777778 & 8 & 0.577775705070475 & 0.577399387702240 \\
& 0.6666666666666667 & 0.652777777777778 & & 0.5777757705070475 & 0.577399387702240 \\
3 & 0.6111111111111111 & 0.598061698654261 & 9 & 0.577529989052300 & 0.577365035376011 \\
& 0.6111111111111111 & 0.598061698654261 & & 0.577529989052300 & 0.577365035376011 \\
4 & 0.591049382716049 & 0.583424704288997 & 10 & 0.577426211591592 & 0.577354708436348 \\
& 0.591049382716049 & 0.583424704288997 & & 0.577426211591592 & 0.577354708436348 \\
5 & 0.583046362978205 & 0.579163300958378 & 11 & 0.577382363341750 & 0.577351603803299 \\
& 0.583046362978205 & 0.579163300958378 & & 0.577382363341750 & 0.577351603803299 \\
6 & 0.579741498953815 & 0.577894163483913 & 12 & 0.577363833259364 & 0.577350670428927 \\
& 0.579741498953815 & 0.577894163483913 & & 0.577363833259364 & 0.577350670428927 \\
\hline
\end{tabular}

Now one can easily compute

$$
\begin{aligned}
\left\|F\left(x_{0}\right)\right\| & =\left\|-1+x_{0}(s)+\pi x_{0}(s) \int_{0}^{1} \frac{s}{s+t} x_{0}(t) d t\right\| \\
& \leq\left\|x_{0}-1\right\|+|\pi| \max _{s \in[0,1]}\left|\int_{0}^{1} \frac{s}{s+t} d t\right|\left\|x_{0}\right\|^{2} \\
& \leq\left\|x_{0}-1\right\|+\pi \log 2\left\|x_{0}\right\|^{2} .
\end{aligned}
$$

Also notice that

$$
\begin{aligned}
\left\|I-F_{x_{0}}^{\prime}\right\| & =\left\|\pi \int_{0}^{1} \frac{s}{s+t} x_{0}(t) d t+\pi x_{0}(s) \int_{0}^{1} \frac{s}{s+t} d t\right\| \\
& \leq 2 \pi \max _{s \in[0,1]}\left|\int_{0}^{1} \frac{s}{s+t} d t\right|\left\|x_{0}\right\| \\
& \leq 2 \pi \log 2\left\|x_{0}\right\|
\end{aligned}
$$

Now, we have

$$
\begin{aligned}
\left\|F_{x}^{\prime}-F_{x_{0}}^{\prime}\right\| & =\left\|\pi \int_{0}^{1} \frac{s}{s+t} x(t) d t+\pi x(s) \int_{0}^{1} \frac{s}{s+t} d t-\pi \int_{0}^{1} \frac{s}{s+t} x_{0}(t) d t-\pi x_{0}(s) \int_{0}^{1} \frac{s}{s+t} d t\right\| \\
& =|\pi| \| \int_{0}^{1} \frac{s}{s+t}\left(x(t)-x_{0}(t) d t+\left(x(s)-x_{0}(s)\right) \int_{0}^{1} \frac{s}{s+t} d t \|\right. \\
& \leq 2 \pi \max _{s \in[0,1]}\left|\int_{0}^{1} \frac{s}{s+t} d t\right|\left\|x-x_{0}\right\| \\
& \leq 2 \pi \log 2\left\|x-x_{0}\right\|
\end{aligned}
$$


and, if $2|\pi| \log 2|| x_{0}||<1$, then by Lemma 2.3, we obtain

$$
\left\|F_{x_{0}}^{\prime}-1\right\| \leq \frac{1}{1-2 \pi \log 2\left\|x_{0}\right\|} .
$$

Hence, we have

$$
\left\|F_{x_{0}}^{\prime}{ }^{-1} F\left(x_{0}\right)\right\| \leq \frac{\left\|x_{0}-1\right\|+\pi|\log 2|\left\|x_{0}\right\|^{2}}{1-2 \pi \log 2\left\|x_{0}\right\|} .
$$

Now, for $\pi=\frac{1}{4}$ and the initial point $x_{0}=x_{0}(s)=1$, we obtain

$$
\|\| F_{x_{0}}^{\prime}{ }^{-1}\|\leq \beta=1.17718382,\| F_{x_{0}}^{\prime}{ }^{-1} F\left(x_{0}\right) \| \leq \eta=0.08859191, K_{0}=0.346573590279973 .
$$

Hence, $h=\beta \eta K_{0}=0.036143800345579<\frac{1}{2}$. So the hypotheses of Theorem 3:5 are satisfied. Therefore, the sequence $\left\{x_{n}\right\}$ generated by Algorithm 1.3 is in $B_{r}\left[x_{0}\right]$ and it converges to a unique solution $x^{*} \in B_{r}\left[x_{0}\right]$ of the integral equation.

\section{Authors' contributions}

All authors contribute equally and significantly in this research work. All authors read and approved the final manuscript.

\section{Competing interests}

The authors declare that they have no competing interests.

Received: 29 October 2011 Accepted: 9 May 2012 Published: 9 May 2012

\section{References}

1. Li, Q, Mo, Z, Qi, L: Numerical Methods for Solving Non-linear Equations. Science Press, Beijing (1997) (in chinese)

2. Argyros, IK: On a class of Newton-like methods for solving nonlinear equations. J Comput Appl Math. 228, 115-122 (2009). doi:10.1016/j.cam.2008.08.042

3. Wu, Q, Zhao, Y: Third-order convergence theorem by using majorizing function for a modified Newton method in Banach space. Appl Math Comput. 175, 1515-1524 (2006). doi:10.1016/j.amc.2005.08.043

4. Argyros, IK: Computational Theory of Iterative Methods, Series: Studies in Computational Mathematics. Elsevier Publ. Comp., New York15 (2007)

5. Bartle, RG: Newton's method in Banach spaces. Proc Am Math Soc. 6, 827-831 (1955)

6. Dennis, JE: On the Kantorovich hypothesis for Newton's method. SIAM J Numer Anal. 6, 493-507 (1969). doi:10.1137/ 0706045

7. Rheinboldt, WC: A unified convergence theory for a class of iterative processes. SIAM J Numer Anal. 5, 42-63 (1968). doi:10.1137/0705003

8. Argyros, IK: Convergence and applications of Newton-type iterations. Springer, Berlin (2008)

9. Ren, H, Argyros, IK: On convergence of the modified Newtons method under Hölder continuous Fréchet derivative. Appl Math Comput. 213, 440-448 (2009). doi:10.1016/j.amc.2009.03.040

10. Agarwal, RP, O'Regan, D, Sahu, DR: Iterative construction of fixed points of nearly asymptotically nonexpansive mappings J. Nonlinear Convex Anal. 8(1):61-79 (2007)

11. Sahu, DR: Applications of the S-iteration process to constrained minimization problems and split feasibility problems. Fixed Point Theory. 12, 187-204 (2011)

12. Scherzer, O: Convergence criteria of iterative methos based on Landweber iteration for solving nonlinear problems. J Math Anal Appl. 194, 911-933 (1995). doi:10.1006/jmaa.1995.1335

13. Ortega, JM, Rheinbolt, WC: Iterative Solution of Nonlinear Equations in Several Variables. Academic Press, New York (1970)

14. Rall, LB: Computational Solution of Nonlinear Operator Equations. Wiley, New York (1969)

15. Agarwal, RP, O'Regan, D, Sahu, DR: Fixed Point Theory for Lipschitzian-type Mappings with Applications. Series: Topological Fixed Point Theory and its Applications. Springer, New York6 (2009)

16. Kreyszig, E: Introductory Functional Analysis with Applications. Wiley, New York (1978)

17. Suhubi, ES: Functional Analysis. Kluwer Academic Publishers, London (2003)

18. Porter, D, Stirling, DSG: Integral Equations. Cambridge University Press, Cambridge (1990)

19. Lambert, JD: Computational Methods in Ordinary Differential Equations. Wiley, New York (1979)

doi:10.1186/1687-1812-2012-78

Cite this article as: Sahu et al:: Some Newton-like methods with sharper error estimates for solving operator equations in Banach spaces. Fixed Point Theory and Applications 2012 2012:78. 